\title{
The Enlightenment of Cognitive Science Theory to Visual Play
}

\author{
Yu Xiaoxin \\ School of Arts, Zhejiang International Studies University, Zhejiang, Hangzhou 310023
}

Keywords: visual play; cognitive science theory; pattern recognition

\begin{abstract}
Various disciplines are using the principles of cognitive science and research results to inspire and direct their own training and research, the same is true for piano sightings. This paper contrasts the pattern recognition theory and information processing theory of cognitive science with visual play, and clarifies the relationship, which in turn inspires the "cognitive process" and concrete training thinking connotation of the visual play itself.
\end{abstract}

With the development of research on cognitive psychology, psychologists have proposed cognitive models, elucidated the mechanisms of psychological development, and produced new ideas on how the brain works. The piano's "cognitive activities," phenomena, understandings, and problems are enough to arouse our thinking.

Based on the above considerations, perhaps the traditional and modern research results of "cognitive psychological science" can guide our thinking, and this paper thus carrys out relevant thinking and exposition, trying to provide some ideas and suggestions for sight-seeing trainers.

\section{Visual Play Training and Pattern Recognition Model}

Various symbols involved in Visual Play are accurately reflected on the keyboard. Based on the following four points, first, the meaning of the symbol itself is stored and stored in the brain; secondly, the corresponding play mode of the symbolic meaning; Third, the key position of the symbol meaning; Fourth, the corresponding sound representation of symbolic meaning.

(1) The meaning expressed by the music notation symbol is fixedly stored in the brain, including the line position, layout, etc.

(2) The corresponding play mode of the symbolic meaning.

The playing action is the reaction of the brain to the arms, fingers, and even the body after processing the musical score symbol.

(3) The key position of the symbol meaning

The key position of the musical notation is the exact "one-to-one correspondence", which is the essence of the fixed spectrum of the line spectrum.

The sight-seeker needs to realize this truth, so as to achieve accurate "one-to-one correspondence" requirements in sight-vision training.

(4) The corresponding sound representation of symbolic meaning.

Piano score symbol sound representation is reflected in two aspects, and these two aspects can appear at the same time, they can also take place in succession. On the one hand, it is the "inner voice" before playing; on the other hand, it is the objective physical sound that is considered after playing. Both voices require comprehensive music training, but it has a role of supervision and judgment in the accuracy of sighting.

The performance of prototype model theory in visual play.

The "correction" performance in the overall feeling

Music, especially traditional music, will have an abstract and specific sound in the overall sense to determine whether the played sound conforms to such content.

The "choice and judgment" of the diverse piano literature itself

There will be differences between the versions of music scores, and it is also the situation often encountered by musicologists when they study composers and works.

"Add" to symbols in visual play 
This "adding" does not mean omission of a sign, but rather a sighting addition similar to a largelength modular cluster.

The performance of distinctive feature model theory in visual play

1) In the face of piano score, the sight-seeker is sometimes overwhelmed. For example, the number of notes between the upper and lower lines, the varying tone level (especially the reload or rewind) on the basis of the basic tone level, and the rest and non-stop are often unrecognizable or incorrect.

Faced with these musical score, each person can use their own habits to compare difficult-toidentify line locations with archetypes and identify "differences" to identify them.

2) The "model" difference between visual and auditory changes. Modifying the sound level is a high error rate in sight. The visualised fears less about music score with fewer lifting or raising marks. In addition to the lack of profound understanding of key numbers and the use of temporary lift marks, there are similar misunderstandings in the visual and auditory aspects that are similar to the "differential feature model".

3) Increase the hand movement model difference. Piano work, notation methods do exist "not strict", resulting in "confusing." Score information does not clearly indicate whether there is an instruction to "raise the hand", but it often requires "lifting the hand and movement". Even the legato will be used in the legato, and the exact details will be different. One of the important techniques is to "raise your hand". As a "model" to distinguish these movements and from "conditional reflexes", it is an important prerequisite for smooth sighting and sighting ability exercises.

\section{The Inspiring Application of Cognitive Psychology to Visual Play}

The most important role of cognitive psychology research methods and results in other disciplines is "inspiration", but also includes reference and extension. Then, how to obtain such "inspiration", "reference" and "extension" for Visual Play is a detailed "visual" analysis of "characteristics of brain cognitive activities" and "concrete steps."

\subsection{Anatomical rhythm reflects the rational meaning - slow speed}

The training of sighting reading must be based on accuracy. Otherwise neither it is conducive to the formation and improvement of it, nor to the formation of good cognitive habits. This requires the "slow speed" of sighting reading. The slow speed emphasizes connotative, operational, rational awareness and subjective control capability.

Firstly, "slowness" should be confirmed as the belief of "absolutely correct." Secondly, "slowness" should not be despised. The slower the more it embodies the rhythm of human perception of time and value. Thirdly, when getting slower and slower, the weaker the sensibility, the stronger the rationality and vice versa.

\subsection{Anatomical methods}

In brief, the anatomical method is the "connotation" to refine and segment the rhythm value. Meanwhile the similarities and commonalities of rhythms in the following examples will be established.

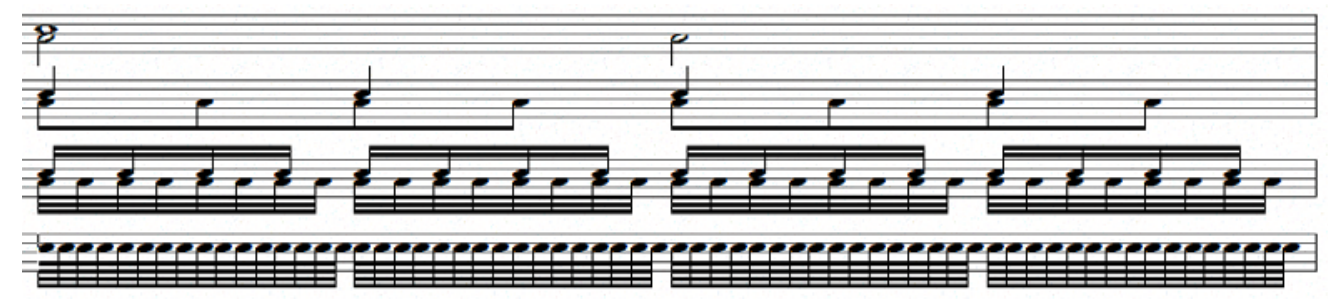

The above is a time-temporal segmentation diagram. Most of the rhythm and its variation of the piano texture can find its own time position (time code) in the above segmentation diagram. This "anatomy" rhythm serves as a support point for slow control. 
The significance of such anatomy is that the musical scores touched by the vision can be clearly "allocated" to sixteen nodes or even thirty-two nodes, so that the smallest "point" is used as the "beat" unit to slowly "move forward”.

\subsection{Anatomical rhythm and will power and brain ability}

Due to the multi-linearity of piano score and the universality of body movements, the theoretical "slow speed" requires patience, attention long-lasting and "high-speed operation" of the brain and so forth. This type of situation is taken into account in teaching and learning practices.

Firstly, be patient and careful. The most direct and simple score information can be summarized as "line pitch position," "all types of pause" "note time value," "various symbols" and so on, which require to be observed carefully and not to be missed to measure the speed of reception and determine "slow speed" value.

Secondly, attention should be long lasting. Although "slow speed" is relatively a "variable value", it tends to develop in a slower direction and thus trains a good "learning quality" named sustained attention.

Thirdly, the brain is "running up" The sight reading practice seems to present a state that is "too late to think". In this sense, the slow speed practice is not only required for sighting reading ability training, but also for learning and improving the ability for "brain drain", and is also the embodiment of the running up of brain during the period of slow speed sighting reading practice.

The meanings represented by symbols such as the center position of piano scores are easy to learn and the information is easy to remember. However, even those who have good flow control ability, in the whole score visual sighting, will encounter the situation that is predicted to be insufficient in advance and cannot slow down to the corresponding speed. At this time, awareness of the pace of tempo and rhythm changes is of great importance.

According to the process of "input, processing, and output" described in the "information processing theory", piano score has a fixed rhythm. When the speed is not suitable during training, errors will occur. The more error reactions occur, the less conducive to the formation of correct cognitive habits and the ability to improve. In this way, it is necessary to use the title of "rhythm and tempo change speed." "Variable speed" is reflected in the "quick change speed" and conscious "gradation speed" in the Visual Play training. In the performance for expressive performance, there will also be speed changes such as gradually getting faster and slower. "graduation speed" of learning and training can be This ability is available during the sight-seeing stage.

\section{Promote Visual Thinking}

In piano playing, information such as position between lines and fingering is the key basic information. In the process of playing, how to grasp the accurate operation of information involves thinking habits and abilities.

The results of cognitive science research show that there are three stages in skills learning: cognition, unity, and self-discipline. Analytical fingerings and their use can explain this problem. Practices that refer only to fix fingering in actual playing do not have analysis and adequate thinking. Therefore, in the teaching practice, students should have an obligation to arrange, analyze, and draw correct results through relevant knowledge of music theory. Only when they are tested, can they correctly play fingering.

\section{The Enrichment of the Teaching Content}

Cognitive science research gives us a large number of clinical cognitive experiments, as well as corresponding results and methods. Inspired by this, we can address any issues that arise in the students"s Visual Plays and propose solutions and methods. The so-called "hands-on-hand" teaching not only corrects its hand type and body type, but also "pulls" on the basis of the students' existing abilities to correctly play notes and tempo. 
Cognitive science has extremely fertile theories and achievements. The pursuit of the cognitive rules and connotations of Visual Plays is conducive to the formation of visual abilities and good thinking habits. In particular, the rational application of cognitive science can subtly cultivate the moral habits and abilities that students observe, analyze, and study.

\section{References}

[1] Fang Ping, Xiong Duanqin, Luo Yi, Cai Hong. "Discussion on the Trend of Cognitive Development Research." "New Exploration of Psychology”. 2000.2

[2] Chen Huanzhi's "Cognitive Psychology", Guangdong Higher Education Press, December 2006, first edition. 